\title{
LETTERS
}

\section{Imaging of Rab5 activity identifies essential regulators for phagosome maturation}

\author{
Masahiro Kitano ${ }^{1,3}$, Michio Nakaya ${ }^{2,4}$, Takeshi Nakamura ${ }^{1}$, Shigekazu Nagata ${ }^{2,4}$ \& Michiyuki Matsuda ${ }^{1}$
}

\begin{abstract}
Efficient phagocytosis of apoptotic cells is crucial for tissue homeostasis and the immune response $\mathrm{e}^{1,2}$. Rab5 is known as a key regulator of the early endocytic pathway ${ }^{3}$ and we have recently shown that Rab5 is also implicated in apoptotic cell engulfment ${ }^{4}$; however, the precise spatio-temporal dynamics of Rab5 activity remain unknown. Here, using a newly developed fluorescence resonance energy transfer biosensor, we describe a change in Rab5 activity during the engulfment of apoptotic thymocytes. Rab5 activity on phagosome membranes began to increase on disassembly of the actin coat encapsulating phagosomes. Rab5 activation was either continuous or repetitive for up to $10 \mathrm{~min}$, but it ended before the collapse of engulfed apoptotic cells. Expression of a dominantnegative mutant of Rab5 delayed this collapse of apoptotic thymocytes, showing a role for Rab5 in phagosome maturation. Disruption of microtubules with nocodazole inhibited Rab5 activation on the phagosome membrane without perturbing the engulfment of apoptotic cells. Furthermore, we found that Gapex5 is the guanine nucleotide exchange factor essential for Rab5 activation during the engulfment of apoptotic cells. Gapex-5 was bound to a microtubule-tip-associating protein, EB1, whose depletion inhibited Rab5 activation during phagocytosis. We therefore propose a mechanistic model in which the recruitment of Gapex-5 to phagosomes through the microtubule network induces the transient Rab5 activation.
\end{abstract}

To make Rab5 activity visible in living cells, we developed a genetically encoded fluorescence resonance energy transfer (FRET) probe, designated Raichu-Rab5. The probe comprised a modified yellow fluorescent protein (YFP) called Venus, the amino-terminal Rab5binding domain of EEA1, a modified cyan fluorescent protein (CFP) called SECFP, and Rab5 (Fig. 1a). In this probe design, an increase in Rab5-GTP results in an increase in FRET, which can be represented by the $525 \mathrm{~nm} / 475 \mathrm{~nm}$ emission ratio. Characterization of Raichu-Rab5 was conducted similarly to that of other Raichu probes reported previously ${ }^{5,6}$. In comparison with the wild-type Rab5 probe, Raichu-Rab5-Q79L—which lacks GTPase activity—had an increased FRET efficiency, whereas Raichu-Rab5-S34N—which shows a reduced affinity for guanine nucleotides- had a decreased FRET efficiency, as expected (Supplementary Fig. 1a). The GTP loading of the Rab5 probes correlated well with that of the authentic Rab5 proteins (Supplementary Fig. 1b), and the GTP loadings obtained here were similar to those reported previously ${ }^{7}$. Next, we examined the sensitivity of Raichu-Rab5 to guanine nucleotide exchange factors (GEFs) and GTPase-activating proteins (GAPs) (Supplementary Fig. 1c). Rab5 GEFs such as Rabex-5, Rin1 and Gapex-5 induced a dose-dependent increase in the FRET efficiency of Raichu-Rab5. In contrast, the expression of Rab5 GAPs such as RabGAP-5 and RN-tre decreased the FRET efficiency in a dose-dependent manner. These results indicate that Raichu-Rab5 is capable of monitoring the balance between GEF and GAP activities towards Rab5.

We then examined the subcellular distribution of Raichu-Rab5. Rab5 localizes at early endosomes and the plasma membrane. Raichu-Rab5 co-localized with authentic Rab5a at endosomal structures in HeLa cells (Supplementary Fig. 2a, top panel). The distribution of Raichu-Rab5 did not overlap with those of Rab7 and Rab11a, which were used as markers for late and recycling endosomes, respectively (Supplementary Fig. 2a, middle and bottom panels). The subcellular distribution of Raichu-Rab5 was very similar to those of early endosomal markers, namely the FYVE domain of EEA1 (ref. 8) and the PX domain of $\mathrm{p}^{\text {phox }}$ (also known as NCF4) ${ }^{9}$ (Supplementary Fig. 2b). Finally, Raichu-Rab5 was found to bind to Rab guanine dissociation inhibitor (RabGDI) as efficiently as authentic Rab5a (data not shown), suggesting that Raichu-Rab5 is also subject to regulation by RabGDI.

By using Raichu-Rab5 we revealed Rab5 activation during the milk-fat-globule epidermal growth factor 8 (MFG-E8)-mediated engulfment of apoptotic cells by Swiss3T3 cells stably expressing integrin $\alpha_{v} \beta_{3}$ (Fig. $1 \mathrm{~b}$ and Supplementary Video 1). The progress of phagocytosis was monitored by phase-contrast images, in which the completion of engulfment was recognizable by the transition of the engulfed apoptotic cells from phase-bright to phase-dark ${ }^{10}$. The accumulation of Raichu-Rab5 around the engulfed apoptotic cells, and an increase in FRET, began during the period of phase shift and reached a peak after the completion of engulfment.

During the course of experiments, we noticed that the abundance of cytoplasmic Raichu-Rab5, probably associated with RabGDI, hampered the quantification of FRET at the membrane. We therefore developed a Raichu-Rab5 probe fused to the carboxy terminus of KiRas protein (Raichu-Rab5/PM), which localized at the plasma membrane and on phagocytic vesicles. This alteration did not affect the activation status of Rab5 probe (Supplementary Fig. 3). With this improved Raichu-Rab5 probe, we examined the time course of Rab5 activation more precisely (Figs 1c and 2e, and Supplementary Video 2). We set the zero time point to be the frame immediately before the initiation of the phase shift, which lasted about 3 min on average. Rab5 activation started during this period of phase shift and reached a peak within $4 \mathrm{~min}$ on average. Very similar results were obtained in a macrophage cell line, BAM3 (Supplementary Fig. 4). The duration of Rab5 activation varied significantly between different phagosomes. In addition, 37\% of phagosomes showed reactivation of Rab5 after the cessation of initial Rab5 activation (Figs 1d and 3c, and Supplementary Video 3). The time course of this Rab5 reactivation also varied between phagosomes: some phagosomes showed a repetitive cycle of activation and inactivation, whereas others showed an abrupt and transient reactivation. 
To gain insight into the role of Rab5 activation in phagocytosis, we examined the precise timing of actin assembly, Rab5 recruitment around phagocytic vesicles, and breakdown of apoptotic thymocytes in Swiss 3T3/integrin $\alpha_{v} \beta_{3}$ cells (Fig. 2). As revealed with enhanced green fluorescent protein (EGFP)-actin, actin fibres could be seen around the phagocytic vesicles from -3 to $2 \mathrm{~min}$, showing that Rab5 is activated during actin disassembly (Fig. 2a, d, e). This observation was shown more directly by simultaneous imaging of monomeric red fluorescent protein (mRFP)-actin and Raichu-Rab5 (Supplementary Fig. 5). Next, accumulation of mEGFP-Rab5 on the phagosome membrane was revealed with $\mathrm{mRFP}$ as a reference of entirely cytosolic protein $^{11}$ (Fig. 2b, f). The ratio of mEGFP-Rab5 versus mRFP increased on phagosomes after 3-7 min of internalization, indicating that Rab5 was recruited to phagosomes during this period. This time course of Rab5 accumulation was similar to that of Rab5 activation. Finally, we examined the timing of apoptotic cell breakdown by the use of EGFPexpressing thymocytes. Owing to the acid-labile nature of EGFP, the collapse of apoptotic cells within phagosomes could be monitored by the extinction of fluorescence. With this method, we found that EGFPexpressing thymocyte fluorescence disappeared during the period from 10 to 20 min (Fig. 2c, g). We confirmed, by the use of a $\mathrm{pH}$ indicator, that the extinction of EGFP fluorescence was correlated with an increase in intracellular pH level (Supplementary Fig. 6). Thus, Rab5 inactivation preceded the decrease in $\mathrm{pH}$ and the breakdown of apoptotic cells. The expression of Rab5-S34N delayed, but did not block, the breakdown of apoptotic thymocytes (Fig. 2h). This indicated that Rab5 activation has a function in phagosome maturation during the engulfment of apoptotic cells, although Rab5-independent pathways may exist. A similar role for Rab5 has been proposed in Fc $\gamma$ R-mediated phagocytosis ${ }^{12,13}$. This is supposed to come from the basic function of Rab5 mediating the fusion of phagosomes with early endosomes ${ }^{14,15}$. Furthermore, active Rab5 is necessary for the recruitment of Rab7 (ref. 13), which regulates the fusion of phagosomes with late endosomes to initiate the lysis of cell corpses.

In the engulfment process, microtubules were required for Rab5 activation. Treatment with $0.1 \mu \mathrm{M}$ nocodazole significantly decreased Rab5 activation at the engulfment site (Fig. 3a), indicating that Rab5 activation depends on the integrity of the microtubule network. When microtubules were stabilized with $10 \mathrm{nM}$ paclitaxel, initial Rab5 activation was observed as in the control cells, but repetitive Rab5 activation was inhibited significantly (Fig. 3b, c). Thus, it seems that the repetitive activation of Rab5 as shown in Fig. 1d is due to the dynamic instability of microtubule plus-ends.

To search for the molecular link between microtubule and Rab5 activation during phagocytosis, candidate Rab5 GEFs were depleted by RNA interference, and the resulting phenotypes were examined. The expressions of Rabex-5, Rin 1 and Gapex-5 were markedly decreased by transfection of the respective short interfering RNA (siRNAs) in Swiss 3T3/integrin $\alpha_{\mathrm{v}} \beta_{3}$ cells (Supplementary Fig. 7a). We then monitored the Rab5 activity during phagocytosis in cells lacking each Rab5 GEF. The depletion of Gapex-5 significantly inhibited Rab5 activation at engulfment sites, whereas neither Rabex-5 depletion nor Rin1 depletion showed any effect on Rab5 activation during phagocytosis (Fig. 3d). Expression of siRNA-resistant Gapex5 restored the Rab5 activation (Fig. 3d and Supplementary Fig. 7c). The involvement of Gapex -5 in Rab5 activation was corroborated by a

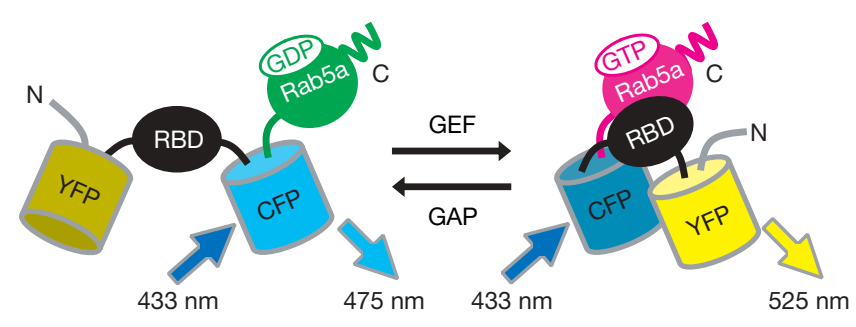

b
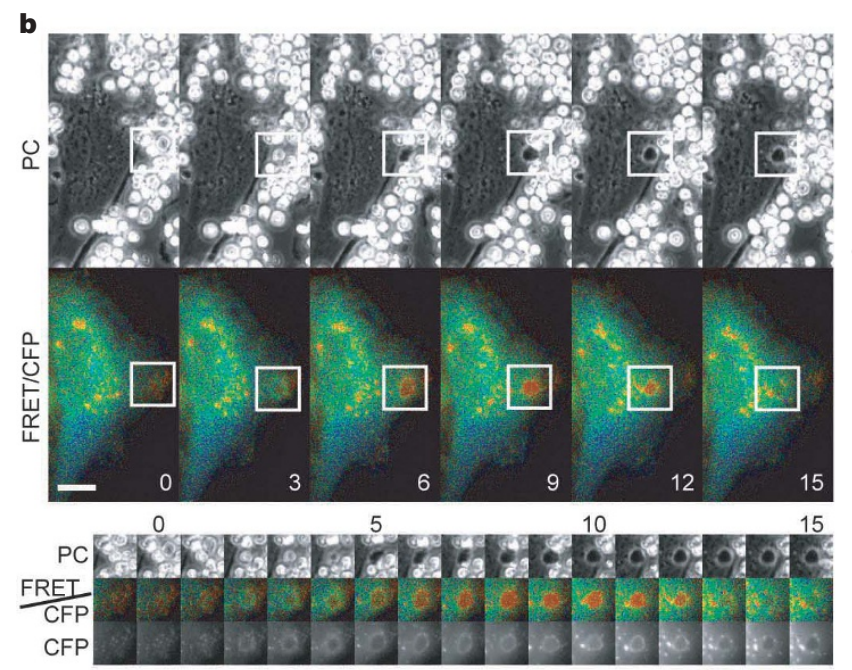

0.51 maแก 0.77

Figure 1 | Transient Rab5 activation during phagocytosis monitored by Raichu-Rab5. a, Schematic representation of Raichu-Rab5 bound to GDP or GTP. b-d, $\alpha_{v} \beta_{3}$ integrin-expressing Swiss3T3 cells were transfected with pRaichu-Rab5 (b) or pRaichu-Rab5/PM (c, d) and cultured together with apoptotic thymocytes in the presence of MFG-E8. Thereafter, images were obtained every $1 \mathrm{~min}$. Top panels show phase-contrast (PC) and FRET/CFP ratio images at the indicated time points $(\mathrm{min})$. In the intensity-modulated c
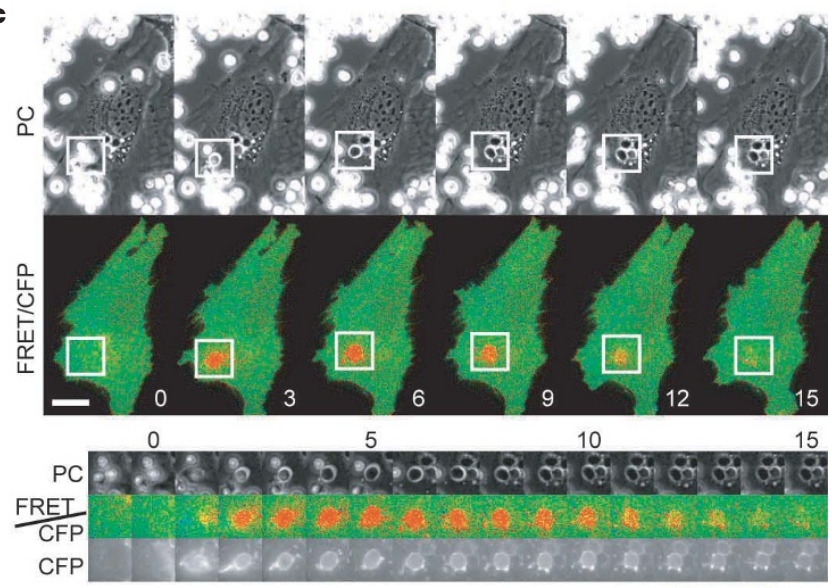

0.54 ำก111 0.81

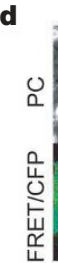
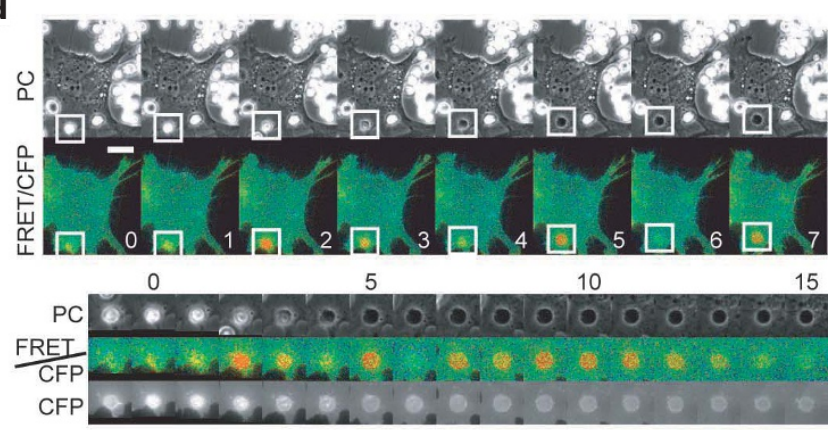

0.53 manm 0.80

display mode shown here, eight colours from red to blue are used to represent the FRET/CFP ratio, with the intensity of each colour indicating the mean intensity of FRET and CFP. The upper and lower limits of the ratio range are shown at the bottom, and the divisions are evenly spaced. Time sequences in the bottom panels show the PC, FRET/CFP ratio and CFP images of the engulfed sites marked by white squares in the top panels. Scale bars, $20 \mu \mathrm{m}$. 
a significant delay in the duration of EGFP-expressing thymocyte fluorescence in Gapex-5-depleted cells (Fig. 3e). These results demonstrated for the first time that Gapex- 5 is an essential regulator for Rab5 activation and phagosome maturation during phagocytosis of apoptotic cells. We assumed that Gapex-5 is delivered to phagosomes by a microtubule-dependent mechanism. This idea could

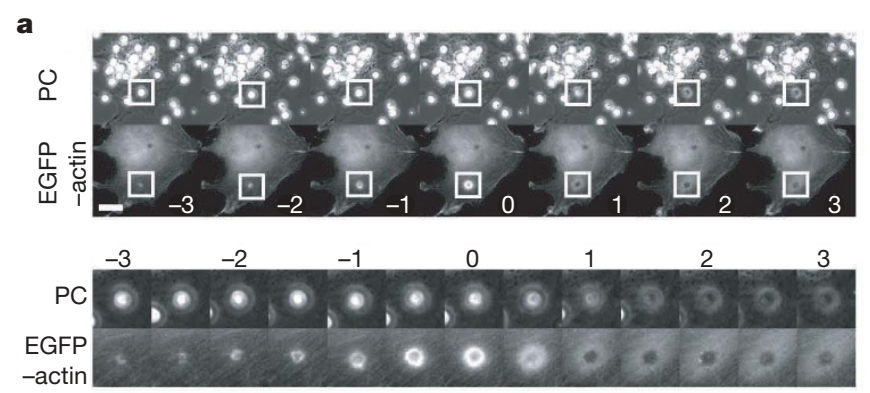

$$
\text { b }
$$
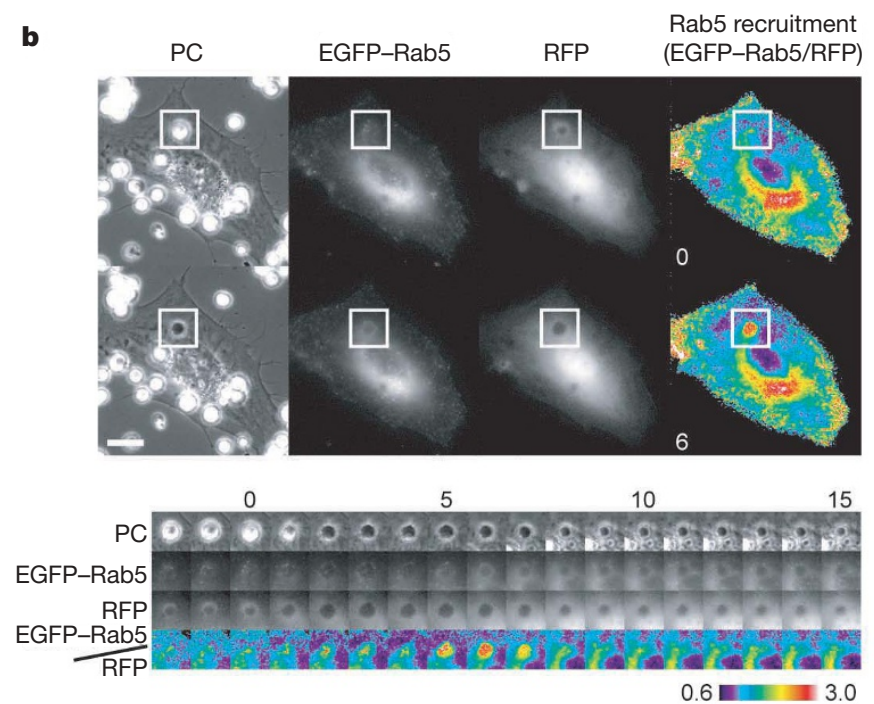

c
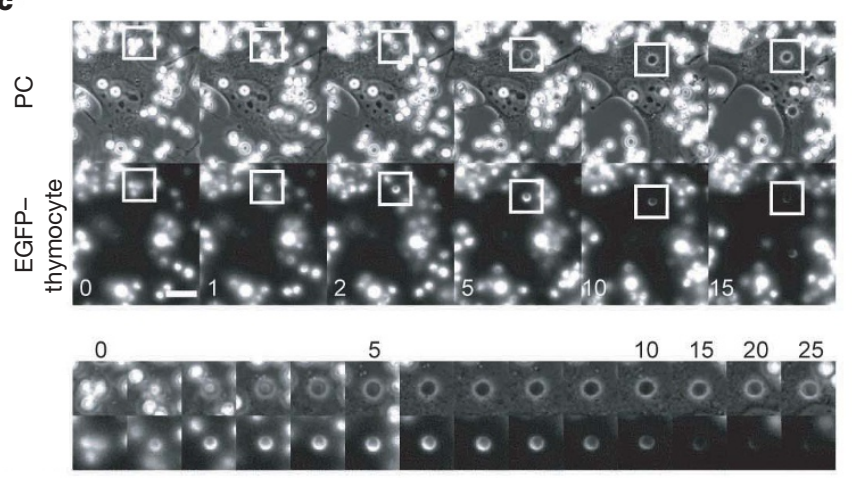

Figure 2 | Time sequence of actin and Rab5 accumulation, Rab5 activation, and breakdown of apoptotic thymocytes. a, b, Swiss3T3/integrin $\alpha_{v} \beta_{3}$ cells expressing EGFP-actin (a) or mEGFP-Rab5 and mRFP (b) were cultured together with apoptotic thymocytes. c, Swiss3T3/integrin $\alpha_{v} \beta_{3}$ cells were cultured together with apoptotic thymocytes derived from green mice. In a and c, the top panels show phase-contrast (PC) and fluorescent images at the indicated time points ( $\mathrm{min}$ ). In the fourth column of $\mathbf{b}$, ratio images of mEGFP-Rab5/mRFP are shown in pseudocolour mode. In a-c, the bottom panels show the images of engulfment sites marked by white squares in the top panels. Scale bars, $20 \mu \mathrm{m}$. d-g, Black lines indicate PC intensity; magenta lines indicate the fluorescence intensity of EGFP-actin (d), the FRET/CFP ratio of Raichu-Rab5/PM (e), the mEGFP-Rab5/mRFP ratio (f), and the fluorescence intensity of EGFP-expressing thymocytes $(\mathbf{g})$ in the circular regions (diameter $7.3 \mu \mathrm{m})$ containing engulfment sites $(n=8$ in each panel). explain the observation that the reduced dynamic instability of microtubules in paclitaxel-treated cells suppressed repetitive Rab5 activation on phagosomes (Fig. 3c). Because Gapex-5 did not show a high affinity for microtubules by itself, we searched for the protein that could link Gapex-5 to microtubule and found that a microtubule-tip-associating protein, EB1 (ref. 16), was associated
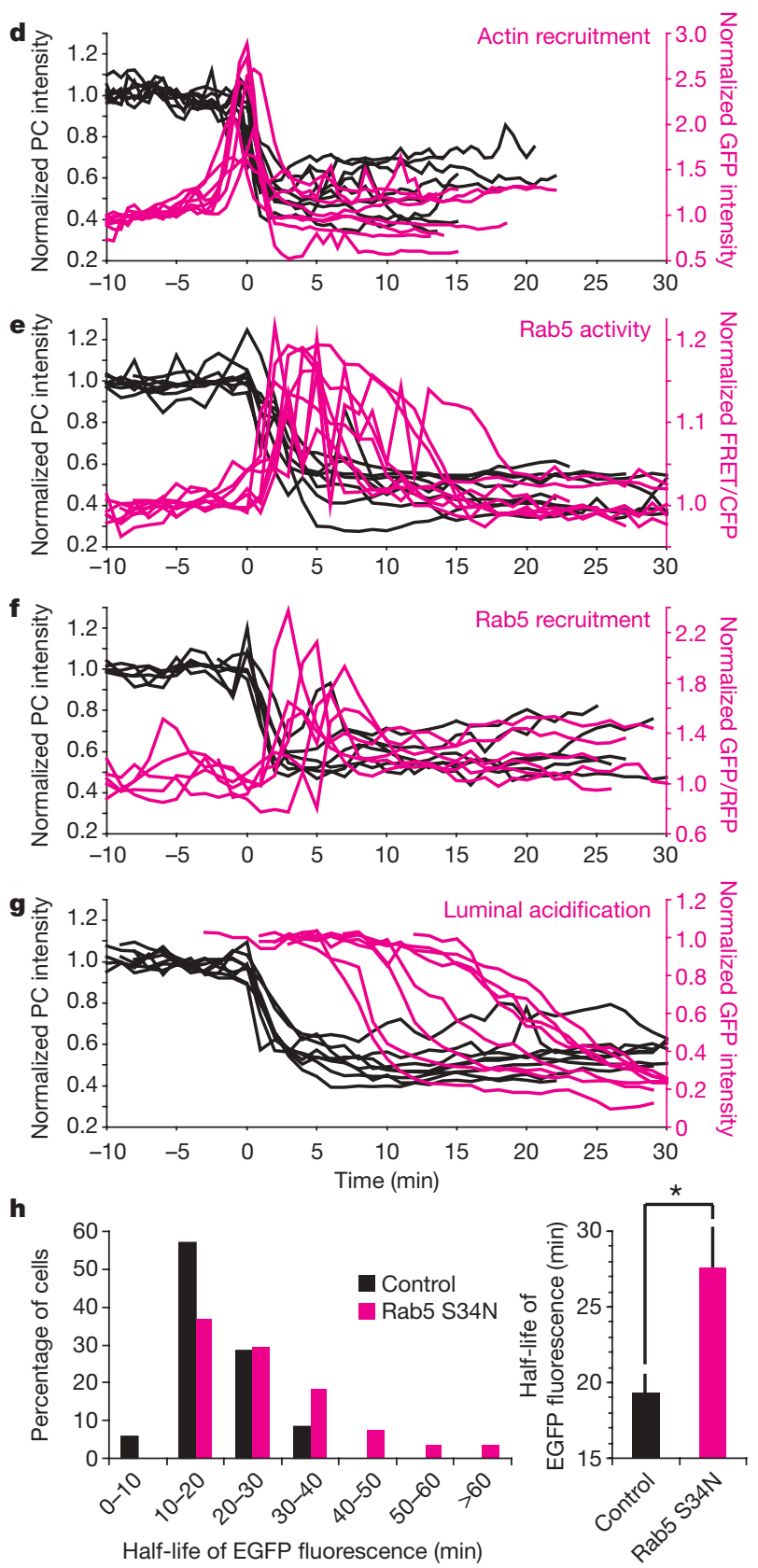

PC intensity $(\mathbf{d}-\mathbf{g})$, EGFP-actin intensity (d), the FRET/CFP ratio (e) and the mEGFP-Rab5/mRFP ratio (f) were normalized by dividing by their average values obtained before zero time (for EGFP-actin intensity (d) the average value between -10 and -5 min was used). EGFP-expressing thymocyte intensity ( $g$ ) was normalized by dividing by the reference value, which was averaged over the period during which the EGFP signal had been stable before the quenching started. h, Control or Rab5-S34N-expressing Swiss3T3/integrin $\alpha_{v} \beta_{3}$ cells were cultured together with apoptotic thymocytes derived from green mice. The half-life of EGFP-expressing thymocyte fluorescence was divided into seven equal segments. Left: histogram plotting the percentage of cell numbers in each segment. Right: half-life of EGFP-expressing thymocyte fluorescence (mean and s.e.m.) $(* P<0.01 ; n>25)$. 

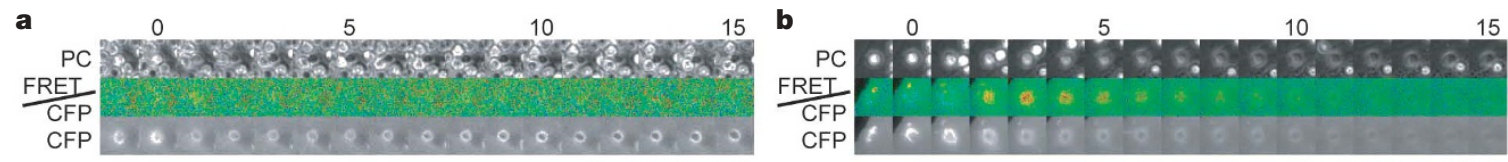

0.54 때111 0.80
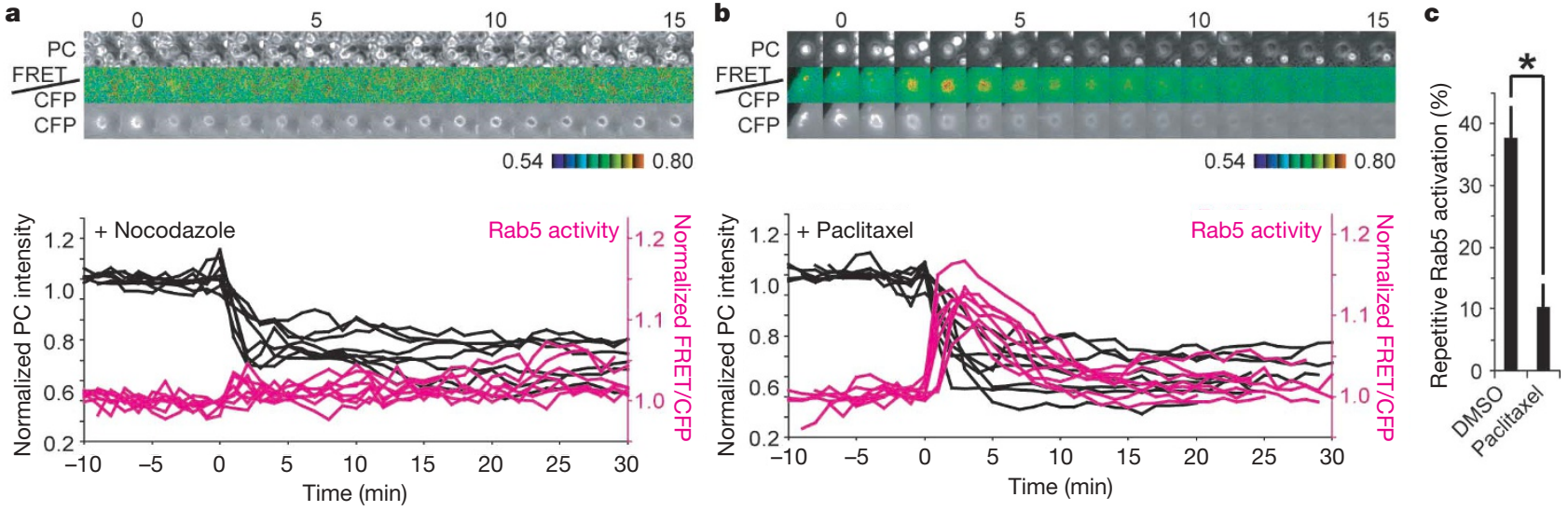

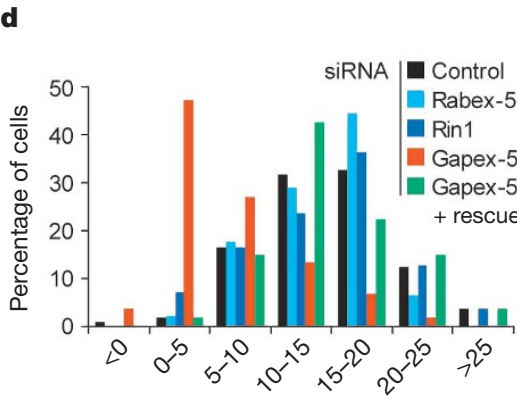

Maximum increase in normalized FRET/CFP (\%)

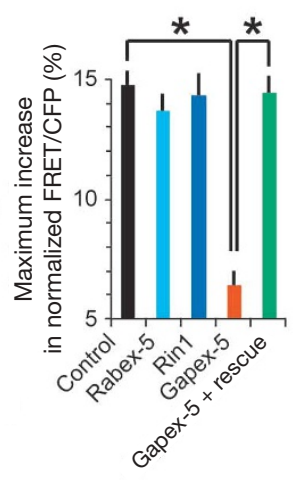

Figure 3 | Involvement of microtubules and Gapex-5 in Rab5 activation at engulfment sites. $\mathbf{a}, \mathbf{b}$, Swiss3T3/integrin $\alpha_{\mathrm{v}} \beta_{3}$ cells expressing RaichuRab5/PM were pretreated with $0.1 \mu \mathrm{M}$ nocodazole (a) or $10 \mathrm{nM}$ paclitaxel (b) for $5 \mathrm{~min}$ and cultured together with apoptotic thymocytes. Time sequences in the top panels show the phase-contrast (PC), FRET/CFP ratio and CFP images at engulfment sites. The bottom panels show the time course of the normalized PC intensity (black) and FRET/CFP ratio (magenta).c, In dimethylsulphoxide (DMSO)-treated and paclitaxel-treated cells, at least 14 events of phagocytosis were observed in each experiment $(n=4)$. The percentage of cells showing reactivation (mean and s.e.m.) is shown $\left({ }^{*} P<0.01\right)$. d, Swiss3T3/integrin $\alpha_{\mathrm{v}} \beta_{3}$ cells treated with siRNA for the indicated Rab5 GEFs were transfected with pRaichu-Rab5/PM and cultured together with apoptotic thymocytes. The maximum relative

with Gapex-5 (Supplementary Fig. 8a, c). In agreement with the role predicted for EB1, EB1 knockdown significantly reduced Rab5 activation during phagocytosis (Supplementary Fig. 8b).

The present study shows that Gapex-5-mediated Rab5 activation follows disassembly of the actin coat surrounding phagosomes. Recalling that the Raichu-Rab5 probe monitors the balance of activity between GEFs and GAPs on the membrane, we envisage the following model for the regulation of Rab5 during phagocytosis: first, engulfment of apoptotic cells and accumulation of actin filaments around the nascent phagosome; second, actin disassembly during phagosome membrane closure; third, invasion of microtubules into the cell periphery (this probably happens because the actin filaments behave as physical barriers for microtubule extension ${ }^{17}$ ); fourth, delivery of Gapex-5 to phagosomes by EB1 on microtubules; fifth, a subsequent increase in the ratio of GEF activity to GAP activity on the phagosome membrane resulting not only in the activation of local Rab5 but also in the recruitment of Rab5 released from the Rab5RabGDI complex in the cytosol. There might be a positive feedback mechanism for local amplification of Rab5 signal, as has been proposed for early endosomes ${ }^{3,18}$. This study shows that live-cell imaging with FRET probes enables us to pinpoint the activation and inactivation of Rab5 and thereby to understand its relationship to the other events of phagocytosis.
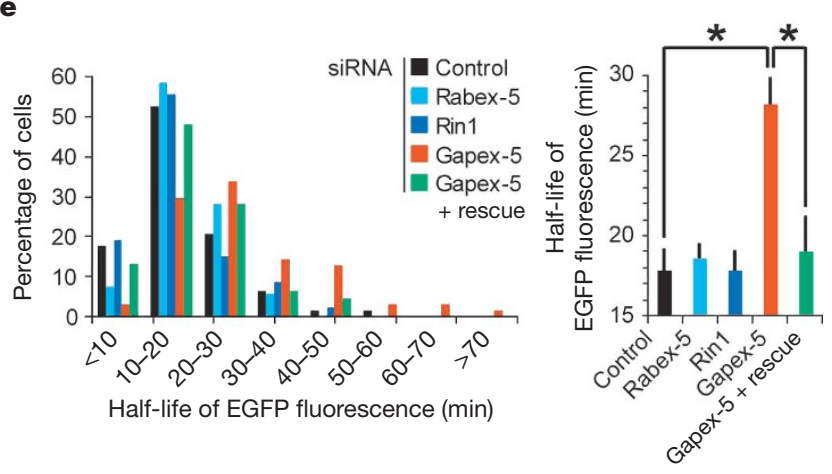

increase in Rab5 activity during phagocytosis was divided into seven segments. A histogram plotting the percentage of cell number in each segment is presented in the left panel. In some experiments we also used Swiss3T3/integrin $\alpha_{v} \beta_{3}$ cells stably expressing Gapex-5 resistant to Gapex-5 siRNA (+ rescue). The Rab5 peak activation (mean and s.e.m.) is shown in the right panel $\left({ }^{*} P<0.001 ; n>40\right)$. e, Swiss3T3/integrin $\alpha_{v} \beta_{3}$ cells treated with siRNA for the indicated Rab5 GEFs were cultured together with apoptotic thymocytes derived from green mice. The half-life of EGFPexpressing thymocyte fluorescence was divided into eight segments. Left: histogram plotting the percentage of cell numbers in each segment. Right: half-life of EGFP-expressing thymocyte fluorescence (mean and s.e.m.) $\left({ }^{*} P<0.001 ; n>40\right)$.

\section{METHODS SUMMARY}

C57BL/6 mice were purchased from Japan SLC. EGFP-transgenic mice ${ }^{19}$ were a gift from M. Okabe. Swiss3T3 fibroblasts stably expressing integrin $\alpha_{\mathrm{v}} \beta_{3}$ were established by infecting cells with a retrovirus carrying mouse integrin $\alpha_{\mathrm{v}}$ and $\beta_{3}$ $\mathrm{CDNAs}^{20}$ and maintained in DMEM medium supplemented with $10 \%$ fetal bovine serum. Retrovirus expressing EGFP-actin, mEGFP-Rab5, mCherryRab5-S34N or mCherry-Gapex-5 resistant to Gapex-5 siRNA was additionally inoculated into Swiss3T3/integrin $\alpha_{\mathrm{v}} \beta_{3}$ cells. Apoptotic thymocytes were prepared as previously described ${ }^{4}$. In brief, thymocytes of 4-8-week-old C57BL/ 6 mice or EGFP-transgenic mice were treated for $5 \mathrm{~h}$ with $10 \mu \mathrm{M}$ dexamethasone at $37^{\circ} \mathrm{C}$ to induce apoptosis. Transient transfection of siRNA was used to decrease the level of specific proteins. For time-lapse FRET imaging, Swiss3T3/integrin $\alpha_{\mathrm{v}} \beta_{3}$ cells expressing FRET probes were cultured in phenol-red-free DMEM/F12 medium supplemented with $10 \%$ fetal bovine serum, and loaded with $4 \times 10^{6}$ apoptotic thymocytes in the presence of $0.1 \mu \mathrm{g} \mathrm{ml}^{-1} \mathrm{MFG}-\mathrm{E} 8$. Cells were imaged with an IX81 inverted microscope (Olympus) equipped with a cooled chargecoupled device (CCD) camera (Retiga Exi, QImaging or Cool SNAP-HQ; Roper Scientific), an IX2-ZDC laser-based autofocusing system (Olympus) and an MD-XY30100T-Meta automatically programmable XY stage (Sigma KOKI). The following filters used for the dual-emission imaging studies were obtained from Omega Optical: an XF1071 (440AF21) excitation filter, an XF2034 (455DRLP) dichroic mirror and two emission filters (XF3075 (480AF30) for CFP and XF3079 (535AF26) for YFP). After background subtraction, FRET/ CFP ratio images were created with MetaMorph software (Universal Imaging), and the images were used to represent FRET efficiency. 
Full Methods and any associated references are available in the online version of the paper at www.nature.com/nature.

\section{Received 9 January; accepted 19 February 2008}

Published online 2 April 2008.

1. Henson, P. M., Bratton, D. L. \& Fadok, V. A. Apoptotic cell removal. Curr. Biol. 11, R795-R805 (2001).

2. Savill, J., Dransfield, I., Gregory, C. \& Haslett, C. A blast from the past: clearance of apoptotic cells regulates immune responses. Nature Rev. Immunol. 2, 965-975 (2002).

3. Zerial, M. \& McBride, H. Rab proteins as membrane organizers. Nature Rev. Mol. Cell Biol. 2, 107-117 (2001).

4. Nakaya, M., Tanaka, M., Okabe, Y., Hanayama, R. \& Nagata, S. Opposite effects of Rho family GTPases on engulfment of apoptotic cells by macrophages. J. Biol. Chem. 281, 8836-8842 (2006).

5. Mochizuki, N. et al. Spatio-temporal images of growth-factor-induced activation of Ras and Rap1. Nature 411, 1065-1068 (2001).

6. Takaya, A., Ohba, Y., Kurokawa, K. \& Matsuda, M. RalA activation at nascent lamellipodia of epidermal growth factor-stimulated Cos7 cells and migrating Madin-Darby canine kidney cells. Mol. Biol. Cell 15, 2549-2557 (2004).

7. Stenmark, H. et al. Inhibition of rab5 GTPase activity stimulates membrane fusion in endocytosis. EMBO J. 13, 1287-1296 (1994)

8. Stenmark, H., Aasland, R., Toh, B. H. \& D'Arrigo, A. Endosomal localization of the autoantigen EEA1 is mediated by a zinc-binding FYVE finger. J. Biol. Chem. 271, 24048-24054 (1996).

9. Kanai, F. et al. The PX domains of p47phox and p40phox bind to lipid products of PI(3)K. Nature Cell Biol. 3, 675-678 (2001)

10. Diakonova, M., Bokoch, G. \& Swanson, J. A. Dynamics of cytoskeletal proteins during Fc $\gamma$ receptor-mediated phagocytosis in macrophages. Mol. Biol. Cell 13, 402-411 (2002).

11. Yoshizaki, H., Mochizuki, N., Gotoh, Y. \& Matsuda, M. Akt-PDK1 complex mediates EGF-induced membrane protrusion through Ral activation. Mol. Biol. Cell 18, 119-128 (2007)

12. Vieira, O. V. et al. Modulation of Rab5 and Rab7 recruitment to phagosomes by phosphatidylinositol 3-kinase. Mol. Cell. Biol. 23, 2501-2514 (2003).
13. Niedergang, F. \& Chavrier, P. Signaling and membrane dynamics during phagocytosis: many roads lead to the phagos(R)ome. Curr. Opin. Cell Biol. 16, 422-428 (2004).

14. Alvarez-Dominguez, C., Barbieri, A. M., Beron, W., Wandinger-Ness, A. \& Stahl, P. D. Phagocytosed live Listeria monocytogenes influences Rab5-regulated in vitro phagosome-endosome fusion. J. Biol. Chem. 271, 13834-13843 (1996).

15. Duclos, S. et al. Rab5 regulates the kiss and run fusion between phagosome and endosomes and the acquisition of phagosomes leishmanicidal properties in RAW 264.7 macrophages. J. Cell Sci. 113, 3531-3541 (2000).

16. Tirnauer, J. S. \& Bierer, B. E. EB1 proteins regulate microtubule dynamics, cell polarity, and chromosome stability. J. Cell Biol. 149, 761-766 (2000)

17. Zhou, F. Q., Waterman-Storer, C. M. \& Cohan, C. S. Focal loss of actin bundles causes microtubule redistribution and growth cone turning. J. Cell Biol. 157 839-849 (2002).

18. Horiuchi, $\mathrm{H}$. et al. A novel Rab5 GDP/GTP exchange factor complexed to Rabaptin-5 links nucleotide exchange to effector recruitment and function. Cell 90, 1149-1159 (1997).

19. Okabe, M., Ikawa, M., Kominami, K., Nakanishi, T. \& Nishimune, Y. 'Green mice' as a source of ubiquitous green cells. FEBS Lett. 407, 313-319 (1997).

20. Hanayama, R. et al. Identification of a factor that links apoptotic cells to phagocytes. Nature 417, 182-187 (2002).

Supplementary Information is linked to the online version of the paper at www.nature.com/nature.

Acknowledgements We thank N. Yoshida, N. Fujimoto, A. Nishiyama, K. Fukuhara, Y. Kasakawa for technical assistance; I. Lodhi and A. Saltiel for unpublished information on Gapex-5; Y. Kiyosue for advice on EB1; and members of the Matsuda laboratory for their input. This work was supported by grants from the Ministry of Education, Culture, Sports, Science, and Technology of Japan.

Author Contributions M.K. and M.N. performed the experimental work and data analysis. T.N., S.N. and M.M. wrote the paper. All authors discussed the results and commented on the manuscript.

Author Information Reprints and permissions information is available at www.nature.com/reprints. Correspondence and requests for materials should be addressed to T.N. (tnakamr@path1.med.kyoto-u.ac.jp). 


\section{METHODS}

Plasmids. pRaichu-Rab5, derived from the pCAGGS expression vector, encoded a probe, designated Raichu-Rab5, that comprised Venus (the brightest version of YFP) ${ }^{21}$, the amino-terminal Rab5-binding domain of EEA1 (amino-acid residues 36-218), SECFP (a brighter version of CFP obtained from A. Miyawaki) and Rab5a (a gift from Y. Takai). In both Venus and SECFP, an A206K mutation was introduced to prevent dimerization between fluorescent protein ${ }^{22}$. In pRaichu-Rab5/PM, the carboxy-terminal peptide of Rab5, QCCSN, was replaced with that of Ki-Ras, RKMSKDGKKKKKKSKTKCVIM. Mouse Rabex- 5 cDNA was cloned from the mouse spleen cDNA library (a gift from N. Mochizuki) and point-mutated to resist siRNA. The cDNA for mCherry was a gift from R. Tsien. mEGFP-Rab5, mCherry-Rab5-S34N (ref. 23) and mCherryGapex-5 resistant to Gapex-5 siRNA were subcloned into pCX4bsr (ref. 24). Retroviral vector pCX4bleo-EGFP-actin was provided by M. Okada.

Mice, cells, reagents and antibodies. C57BL/6 mice were purchased from Japan SLC. EGFP-transgenic mice ${ }^{19}$ were a gift from M. Okabe. Swiss3T3 fibroblasts stably expressing integrin $\alpha_{\mathrm{v}} \beta_{3}$ were established by infecting cells with a retrovirus carrying mouse integrin $\alpha_{\mathrm{v}}$ and $\beta_{3} \mathrm{cDNAs}^{20}$ and were maintained in DMEM medium supplemented with $10 \%$ fetal bovine serum. Retrovirus expressing EGFP-actin, mEGFP-Rab5, mCherry-Rab5-S34N or mCherry-Gapex-5 resistant to Gapex-5 siRNA was additionally inoculated into Swiss3T3/integrin $\alpha_{v} \beta_{3}$ cells. For imaging experiments, cells were plated on fibronectin-coated 35mm glass-base dishes (Asahi Techno Glass).

Preparation of apoptotic thymocytes. Apoptotic thymocytes were prepared as described previously ${ }^{4}$. In brief, thymocytes of 4-8-week-old C57BL/6 mice or EGFP-transgenic mice were treated with $10 \mu \mathrm{M}$ dexamethasone at $37^{\circ} \mathrm{C}$ for $5 \mathrm{~h}$ to induce apoptosis.

RNA interference experiments. Swiss3T3/integrin $\alpha_{v} \beta_{3}$ cells were transfected with the desired siRNAs by using Lipofectamine RNAiMAX (Invitrogen). siRNA sequences for control, mouse Rabex-5, mouse Rin 1 and mouse Gapex-5 were 5' CACCUAAUCCGUGGUUCAA-3', 5' -UUUAUAGAGACGCGUCAUGAUGUGC-3', 5'-UUAUACAUUUGCUUCACACCUAAGC-3' and 5'-GGAGUGUGAUGAAUCGGAUCUUCAA-3', respectively. After $24 \mathrm{~h}$, the cells were treated with trypsin and plated on glass-base dishes. For FRET imaging, the cells were then transfected with pRaichu-Rab5/PM. Thereafter, the cells were incubated for at least $72 \mathrm{~h}$ after siRNA transfection before imaging experiments.

Time-lapse FRET imaging. Swiss3T3/integrin $\alpha_{v} \beta_{3}$ cells expressing FRET probes were cultured in phenol-red-free DMEM/F12 medium supplemented with $10 \%$ fetal bovine serum, and loaded with $4 \times 10^{6}$ apoptotic thymocytes in the presence of $0.1 \mu \mathrm{g} \mathrm{ml}^{-1}$ MFG-E8. Cells were imaged with an IX81 inverted microscope (Olympus) equipped with a cooled CCD camera (Retiga Exi, QImaging or Cool SNAP-HQ; Roper Scientific), an IX2-ZDC laser-based autofocusing system (Olympus) and an MD-XY30100T-Meta automatically programmable $X Y$ stage (Sigma KOKI). The following filters used for the dualemission imaging studies were obtained from Omega Optical: an XF1071 (440AF21) excitation filter, an XF2034 (455DRLP) dichroic mirror, and two emission filters (XF3075 (480AF30) for CFP and XF3079 (535AF26) for YFP). After background subtraction, FRET/CFP ratio images were created with MetaMorph software (Universal Imaging), and the images were used to represent FRET efficiency.

21. Nagai, T. et al. A variant of yellow fluorescent protein with fast and efficient maturation for cell-biological applications. Nature Biotechnol. 20, 87-90 (2002).

22. Zacharias, D. A., Violin, J. D., Newton, A. C. \& Tsien, R. Y. Partitioning of lipidmodified monomeric GFPs into membrane microdomains of live cells. Science 296, 913-916 (2002).

23. Imamura, H. et al. Rho and Rab small G proteins coordinately reorganiza stress fibers and focal adhesions in MDCK cells. Mol. Biol. Cell 9, 2561-2575 (1998).

24. Akagi, T., Sasai, K. \& Hanafusa, H. Refractory nature of normal human diploid fibroblasts with respect to oncogene-mediated transformation. Proc. Natl Acad. Sci. USA 100, 13567-13572 (2003). 